\title{
Efecto de las TIC en el rendimiento educativo: el Programa Conectar Igualdad en la Argentina
}

\section{Maria Verónica Alderete y María Marta Formichella}

RESUMEN

El objetivo de este trabajo consiste en determinar cuál es el "premio", en términos de rendimiento educativo, de los estudiantes beneficiados con el Programa Conectar Igualdad con respecto a aquellos que no participaron en él. Con este fin, se emplea la técnica de emparejamiento o método de PSM (propensity score matching). Se describen los antecedentes del Programa, así como el marco teórico con que se definen los factores explicativos del rendimiento educativo y que posiblemente inciden en la probabilidad de participar en el Programa. La población bajo estudio son los estudiantes de 15 años de la Argentina. Se emplean datos del Programa Internacional de Evaluación de Estudiantes (PISA) correspondientes al año 2012. De acuerdo con los resultados obtenidos, existen diferencias estadísticamente significativas en el rendimiento educativo promedio derivadas de la participación en el Programa Conectar Igualdad. de acción, evaluación, Argentina IIEss (Consejo de Investigaciones Científicas y Técnicas (conicet), Universidad Nacional del Sur-Uns), Departamento de Economía (UNs).mvalderete@iiess-conicet.gob.ar.

María Marta Formichella es Investigadora del Instituto de Investigaciones Económicas y Sociales del Suriıess (Consejo de Investigaciones Científicas y Técnicas (conicet), Universidad Nacional del Sur-uns), Departamento de Economía (uns).mformichella@iiess-conicet.gob.ar. 


\section{I}

\section{Introducción}

Durante las últimas décadas, los sistemas educativos han sido partícipes de los importantes cambios asociados a la difusión de las nuevas tecnologías de la información y la comunicación (TIC) a nivel mundial. La multiplicación exponencial de la información disponible para cualquier individuo en cualquier lugar y el acceso e intercambio de esta independientemente de la presencia física de los usuarios, han transformado la manera en que las personas trabajan, se organizan, socializan, crean, participan del espacio público y usan su tiempo libre (Castells, 1999, en Claro y otros, 2011).

La incorporación de las TIC ocupa actualmente un lugar muy significativo entre las prioridades educativas. Estas tecnologías pueden contribuir al acceso universal a la educación, la igualdad en la instrucción, el ejercicio de la enseñanza, el aprendizaje de calidad y el desarrollo profesional de los docentes, así como a la gestión, dirección y administración más eficiente del sistema educativo. En consecuencia, su aporte es fundamental para el logro de sociedades con mayor igualdad (UNESCO, 2014).

El análisis de las TIC en el sector de la educación está muy ligado a los objetivos de calidad, equidad y eficiencia (Sunkel y Trucco, 2012). Su integración en el ámbito de las instituciones educativas implica volver a pensar tanto la configuración institucional como las prácticas que de ella derivan. Asimismo, requiere reflexionar sobre el desarrollo curricular-escolar y el trabajo de docentes y alumnos en las aulas (Consejo Federal de Educación, 2010).

Por otra parte, la repercusión de las TIC se evidencia en la agenda de políticas educativas de cada uno de los países latinoamericanos (SITEAL, 2014), en los que se han implementado diversos programas de incorporación

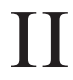

\section{Estado del arte}

Las TIC extienden las oportunidades de comunicación en las instituciones educativas y fuera de ellas, generando nuevas oportunidades que impulsan a los estudiantes al aprendizaje y a la inclusión de aquellos estudiantes a los masiva de las TIC. Estos modelos no son homogéneos sino que, por el contrario, presentan diferentes modalidades en cada contexto nacional. En la Argentina se destaca el Programa Conectar Igualdad como una forma de política de inclusión digital de alcance federal.

La disponibilidad de información a nivel nacional en torno del uso de las TIC en la educación media no es exhaustiva. Solo en un reducido grupo de países latinoamericanos (Chile, Panamá, Trinidad y Tabago y Uruguay) se aplicó el cuestionario TIC complementario del Programa Internacional de Evaluación de Estudiantes (PISA, por sus siglas en inglés) y la Argentina no forma parte de este.

El objetivo del presente trabajo es determinar cuál es el "premio", en términos de rendimiento educativo, de los estudiantes beneficiados con el Programa Conectar Igualdad respecto de aquellos no participantes. Con dicho fin, se utiliza la técnica de emparejamiento o método de PSM, empleando los datos de la Prueba PISA para la Argentina correspondientes al año 2012. El trabajo se estructura de la siguiente manera. En la sección II se plantea un estado del arte de la investigación en torno del papel de las TIC en la educación y se ofrece un marco de las iniciativas de implementación de las TIC, especialmente en países de América Latina, con énfasis en el caso de la Argentina. En la sección III se presenta el marco analítico que describe los hallazgos teóricos y empíricos alcanzados respecto de la repercusión de las TIC en el rendimiento educativo. En la cuarta sección se explica la metodología de PSM aplicada y se detallan los datos y variables utilizados. Por último, en la quinta sección se esbozan los resultados y se presentan las consideraciones finales. que el sistema educativo formal no puede dar cobertura (Becta, 2007). La participación en la sociedad de la información implica no solo tener acceso a las nuevas tecnologías, sino también desarrollar las capacidades 
de uso necesarias. De este modo, las TIC constituyen un importante instrumento para fomentar las prácticas inclusivas (Claro, 2011).

El acceso a las TIC en el sector educativo se relaciona con la disponibilidad de recursos materiales en un establecimiento de enseñanza (Sunkel y Trucco, 2012). Las computadoras permiten lograr reducciones de tiempo y costos, velocidad en los resultados, aprendizaje a distancia, medición del aprendizaje de los estudiantes mediante la recolección de los puntajes obtenidos en los exámenes y el monitoreo de los avances logrados en clases, entre otras ventajas (Witte y Rogge, 2014; Terzis y Economides, 2011; Parshall y otros, 2002).

Por otra parte, mediante el uso de las TIC, la capacitación y el aprendizaje pueden estar al alcance de un gran número de personas a un costo marginal bajo. Los ahorros en viajes y las economías de escala que se obtienen reducen los costos de aprendizaje y permiten alcanzar costos eficientes (Maguire y Zhang, 2007).

Las nuevas TIC permiten un acceso más rápido y eficaz de docentes y estudiantes a la información, reduciendo de este modo el grado de obsolescencia de la información, y utilizando de forma más eficiente las distintas fuentes informativas existentes a través de la red (Lara y Duart, 2005).

Cabe señalar que el uso eficaz de las TIC para la enseñanza y el aprendizaje va a depender, en gran medida, de la actitud de los equipos directivos y de los docentes, quienes pueden garantizar que el acceso sea acompañado de un uso adecuado de los recursos. Sin embargo, el mayor interés - tanto en la Argentina como en otros países - se encuentra en el uso educativo de las computadoras, o Educación basada en las Computadoras (enseñanza asistida por ordenador), que en inglés se conoce como CAI (computer aided instruction), más que en la capacitación en el uso de las computadoras o CST (computer skill training) (Angrist y Lavy, 2002).

En América Latina, la Comisión Económica para América Latina y el Caribe (CEPAL) lleva a cabo el proyecto "Alianza para la Sociedad de la Información 2 - Diálogo político inclusivo e intercambio de experiencias, @LIs2”, que se desarrolla en conjunto con la Unión Europea. En este proyecto se sostiene que las TIC no son un fin en sí mismas, sino solo un medio para lograr objetivos de desarrollo, y —en al ámbito educativo - para lograr objetivos educacionales.

En el Plan de Acción sobre la Sociedad de la Información de América Latina y el Caribe (elAC 2010), donde se define un conjunto de estrategias que promueven el uso de las TIC con miras al desarrollo, se ha identificado a la educación como la prioridad para el desarrollo con equidad en la sociedad de la información (Sunkel y Trucco, 2010).

Asimismo, el Banco Mundial ha formulado estrategias de apoyo a los países de América Latina para aprovechar las oportunidades de las TIC en la educación, brindando equipos e instalaciones, capacitación a docentes, aprendizaje a distancia, alfabetización digital y evaluación, entre otros. En igual sentido, el Banco Interamericano de Desarrollo (BID) favorece iniciativas en que estas tecnologías se incorporen en la educación para mejorar la calidad de aprendizaje de los estudiantes (Claro, 2011).

Los esfuerzos realizados hasta ahora por los sistemas educativos en América Latina han tenido como principal énfasis dotar equitativamente a las escuelas de una infraestructura tecnológica adecuada (especialmente computadoras e Internet) y alfabetizar a los estudiantes y profesores en algunos de sus usos básicos (Sunkel, Trucco y Möller, 2011).

Claro y otros (2011) muestran que los sistemas escolares de la región han logrado compensar en parte las desigualdades de acceso a las TIC en el hogar, ofreciendo amplia cobertura en las escuelas. A pesar de esto, la posibilidad de un uso efectivo de las TIC de manera frecuente sigue siendo mayor en los hogares que en las instituciones educativas debido, por una parte, a la insuficiente cantidad de computadoras con respecto al número de alumnos, y por otra, a la falta de conexión a Internet. Entre los usos más frecuentes de las TIC en ambos casos se halla el apoyo a las tareas escolares.

Se observa que la Argentina perdió liderazgo en los últimos años y ocupa el tercer lugar con relación al acceso a computadoras, que es encabezado por el Uruguay y Chile. Con respecto al acceso a Internet, que lideran el Brasil, el Uruguay y Chile, la Argentina ocupa el cuarto lugar (véase el gráfico 1).

Según datos proporcionados por PISA 2009, la brecha entre América Latina y la Organización para la Cooperación y el Desarrollo Económicos (OCDE) en el porcentaje de estudiantes de 15 años con acceso a computadoras es muy similar a la que existe en el acceso a Internet (alrededor del doble) y se ha sostenido en el tiempo. Asimismo, todavía una buena parte de los jóvenes de la región no cuenta con tecnología en sus hogares. No obstante, se ha avanzado en lo que se refiere al número de alumnos por computadora y al porcentaje de computadoras con acceso a Internet en los establecimientos educativos (Claro y otros, 2011).

En los países de América Latina se han implementado diversos programas de incorporación masiva de TIC con diferentes modalidades. En el cuadro 1 pueden observarse los programas implementados en algunos países de América Latina. 
GRÁFICO

América Latina y el Caribe (siete países): porcentaje de estudiantes de 15 años con acceso a computadora e Internet en el hogar, 2000 a 2009

A. Con acceso a computadora

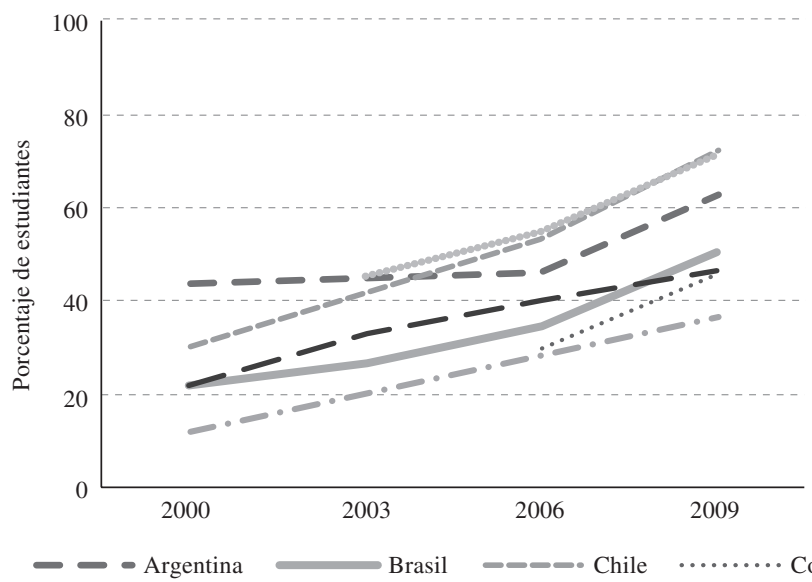

B. Con acceso a Internet

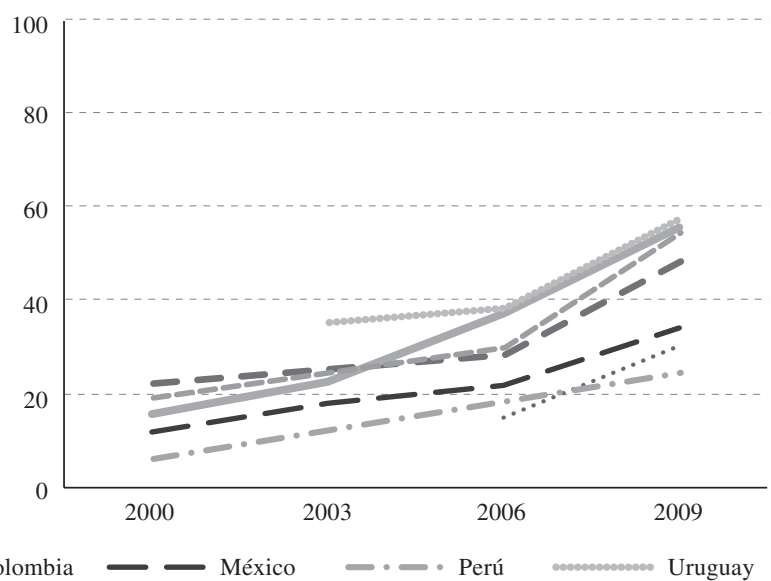

Fuente: M. Claro y otros, "Aporte del sistema educativo a la reducción de las brechas digitales. Una mirada desde las mediciones PISA", Documento de Proyecto (LC/W.456), Santiago, Comisión Económica para América Latina y el Caribe (CEPAL), 2011.

CUADRO 1

Programas sobre las TIC y educación en América Latina

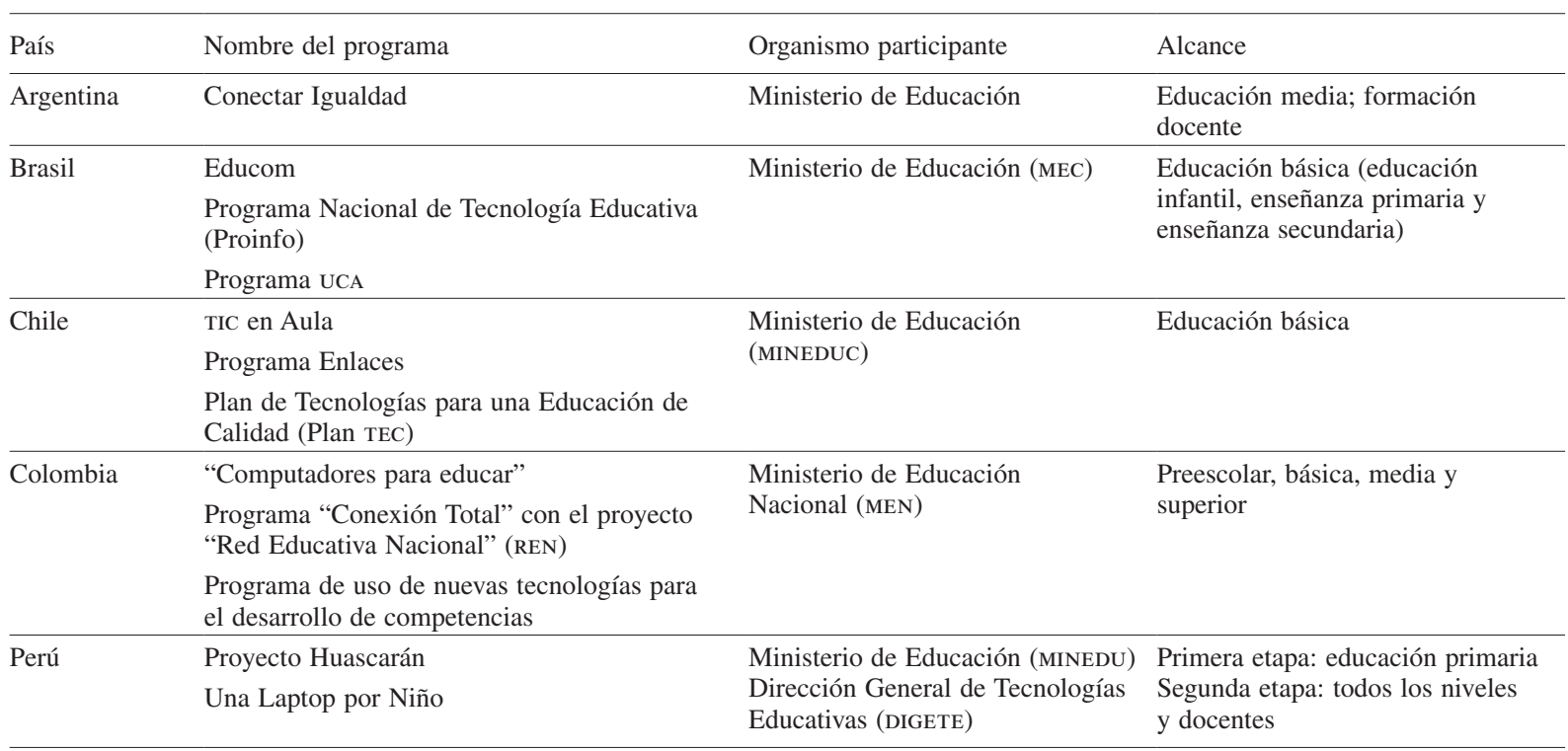

Fuente: Elaboración propia sobre la base de Fondo de las Naciones Unidas para la Infancia (UNICEF), Las políticas TIC en los sistemas educativos de América Latina. Caso Brasil. Programa TIC y Educación Básica, Buenos Aires, 2014; Las políticas TIC en los sistemas educativos de América Latina. Caso Colombia. Programa TIC y Educación Básica, Buenos Aires, 2014; Las políticas TIC en los sistemas educativos de América Latina: caso argentino, Buenos Aires, 2013; Las políticas TIC en los sistemas educativos de América Latina. Caso Chile. Programa TIC y Educación Básica, Buenos Aires, 2013; Las políticas TIC en los sistemas educativos de América Latina. Caso Perú. Programa TIC y Educación Básica, Buenos Aires, 2013. 
En la Argentina, la Ley de Educación Nacional $\left(\mathrm{N}^{\circ}\right.$ 26.206) incorpora la temática de las TIC como disposición de la política educativa nacional, de promoción de la igualdad educativa y de la calidad de la educación. La relevancia política de una escuela que favorezca la integración de las TIC queda explicitada en dicha ley al plantear en sus artículos 7 y 8 que "el Estado garantiza el acceso de todos/as los/las ciudadanos/as a la información y al conocimiento como instrumento central de la participación en un proceso de crecimiento económico y justicia social" y que "la educación brindará las oportunidades necesarias para desarrollar y fortalecer la formación integral de las personas a lo largo de toda la vida y promover en cada educando/a la capacidad de definir su proyecto de vida, basado en los valores de libertad, paz, solidaridad, igualdad, respeto a la diversidad, justicia, responsabilidad y bien común". En los últimos años, el trabajo con las TIC en las escuelas argentinas ha ido aumentando de modo evidente ${ }^{1}$.

El Programa Conectar Igualdad fue creado en abril de 2010 mediante el Decreto $N^{\circ} 459 / 10$ con el fin de reducir las brechas digitales, educativas y sociales en la Argentina. Es decir, surge como una iniciativa de política de inclusión digital de alcance federal y es gestionado por la Administración Nacional de la Seguridad Social (ANSES), el Ministerio de Planificación Federal, Inversión Pública y Servicios, y la Jefatura de Gabinete de Ministros.

Conectar Igualdad está destinado a garantizar el acceso y uso de las TIC mediante la distribución de computadoras portátiles a todos los alumnos y docentes de las escuelas secundarias, de educación especial y de los institutos de formación docente de gestión estatal. Asimismo, uno de sus objetivos generales es garantizar la infraestructura de un piso tecnológico básico que

1 En la Argentina se han adoptado iniciativas de integración de computadoras y otros dispositivos, de dotación de conectividad, de capacitación docente y de elaboración de recursos digitales desde los años noventa, con distintos alcances y resultados. Si bien fueron importantes para acercar a la comunidad educativa a las TIC, significaron un moderado avance con niveles no satisfactorios en cuanto a cobertura, capacitación y recursos educativos (UNICEF, 2013a). permita: el aprovechamiento de la conectividad de manera extensiva, la instalación de redes y el uso en las aulas de una computadora por alumno. Este objetivo es responsabilidad del Ministerio de Planificación Federal, Inversión Pública y Servicios.

Para el cumplimiento de los objetivos mencionados se ha definido una distribución gradual de los netbooks, habiéndose alcanzado a noviembre de 2014 la entrega de más de cuatro millones y medio (ANSES, 2014). Por último, cabe señalar que el programa contempla el uso de los netbooks tanto en las escuelas como en los hogares de los alumnos y docentes.

Para comprender la capacidad endógena de innovación en tecnología en la Argentina, así como en otros países periféricos, es preciso analizar la dinámica de los diferentes actores sociales involucrados en los proyectos: el Estado en sus diferentes niveles, las instituciones educativas, los directivos y docentes, las familias, las empresas del sector de las TIC y los ciudadanos en general. Según los contextos y el momento histórico que se desarrolle, pueden existir tensiones entre las lógicas de cada uno de ellos (UNICEF, 2013a). En tal sentido, el Programa Conectar Igualdad, como política de Estado, permite orientar y establecer la agenda del conjunto de las instituciones y actores públicos y privados que intervienen. A su vez, el Programa integra el Plan Nacional Argentina Conectada, que articula a las distintas políticas públicas en relación con las TIC que se encuentran en ejecución en la esfera del Poder Ejecutivo Nacional.

Una característica de la complejidad del Programa Conectar Igualdad es que posee objetivos múltiples, pero con una clara apuesta por ser parte de los procesos de mejoramiento de la calidad educativa en la Argentina (UNICEF, 2013a). A fin de que el Programa cumpla con sus objetivos de garantizar una educación de calidad y la incorporación de las TIC a los procesos de enseñanza y aprendizaje, las netbooks poseen una gran cantidad de recursos educativos, diseñados en su gran mayoría por el portal Educ.ar, así como software educativo instalado, tanto para los sistemas operativos Windows como para Linux. 


\section{III}

\section{Antecedentes de las TIC y rendimiento educativo}

La investigación a nivel internacional evidencia que para mejorar los logros educativos de los estudiantes, no basta con que el centro escolar provea el acceso a las TIC, sino que este debe ser capaz de entregar oportunidades reales de uso y adecuada calidad en el acceso a ellas (Selwyn, 2004). La oportunidad de uso se refiere a la posibilidad real de contacto de los estudiantes con la tecnología, lo que depende de la cantidad de computadoras disponibles y con acceso a Internet, entre otras cuestiones. Por una parte, la calidad del acceso se relaciona con la facilidad, velocidad y fluidez para operar la tecnología disponible. Se mide con indicadores como la velocidad real de la conexión a Internet, la facilidad para realizar operaciones de encendido, el intercambio de información y respaldo de datos personales, entre otros. Asegurar que las TIC estén disponibles de manera equitativa resulta una condición básica para reducir la brecha de acceso o primera brecha digital (Claro y otros, 2011).

Por otra parte, el aprovechamiento de las TIC por un estudiante no solo depende de las oportunidades disponibles, sino también del tipo de actividad realizada mediante las nuevas tecnologías en el centro escolar. Se deben crear e implementar modelos de aprendizaje que desarrollen habilidades cognitivas y permitan un uso educativamente relevante. Esto conduce a la segunda brecha digital que no se refiere a las diferencias de acceso, sino a las diferencias en el uso de las TIC y la capacidad de beneficiarse de ellas (Hargittai, 2002; Robinson, DiMaggio y Hargittai, 2003).

Las TIC han sido introducidas en las escuelas para transformar los procesos de enseñanza y aprendizaje y mejorar las estrategias con miras al logro educativo (Kozma, 2003 y 2008; Sunkel, 2006; Carneiro, Toscano y Díaz, 2009; Rodríguez, Nussbaum y Dombrovskaia, 2013). Por ello, diversos trabajos se han dedicado a investigar el efecto de las mismas sobre el rendimiento escolar (Machin, McNally y Silva, 2006; Aristizabal, Caicedo y Escandón, 2009; Spiezia, 2010; Carrillo, Onofa y Ponce, 2010; Cristia y otros, 2012). Asimismo, en la literatura también se dispone de estudios que, si bien han centrado la atención en otros determinantes de los resultados educativos, han utilizado variables referidas a las TIC como control.

El estudio de los determinantes del rendimiento educativo comenzó en la década de 1960 con el informe
Coleman (Coleman y otros, 1966). A partir de allí, en una gran cantidad de investigaciones se han estudiado los factores personales, familiares y escolares que inciden en los logros educativos (véanse Formichella, 2011 o Formichella y Krüger, 2013 para una revisión detallada).

Aristizabal, Caicedo y Escandón (2009) analizan los factores que influyen en los logros escolares, medidos de acuerdo con la prueba PISA 2006 y 2009, en Colombia. Con ese fin proponen un modelo de ecuaciones estructurales, que les permite analizar relaciones recíprocas y simultáneas entre las variables. Así encuentran que tanto las TIC del hogar (uso de Internet y sus herramientas, y otros dispositivos como consolas de juegos) como las escolares (computadora y software educacional) inciden positivamente en el rendimiento de los estudiantes, siendo mayor la contribución de estas últimas.

Sin embargo, Spiezia (2010) — quien analiza el impacto de las TIC en los resultados educativos promedio con el programa PISA 2006 para el total de los países participantes - destaca el papel del uso de las tecnologías en los hogares de los estudiantes. Este autor constata que existe un efecto significativo en el rendimiento escolar y que este, en la mayoría de los países, es más significativo si la computadora es utilizada en el hogar, por sobre el uso en la escuela. En consecuencia, cuestiona aquellas políticas que proponen incorporar el uso de computadoras como herramienta de aprendizaje en el ámbito escolar.

En igual sentido, Machin, McNally y Silva (2006) se enfocan en el caso de Inglaterra y analizan el impacto causal del gasto destinado a financiar las TIC en los resultados educativos de los estudiantes pertenecientes a diferentes distritos. Estos autores encuentran evidencia en favor de este efecto en el rendimiento en inglés y en ciencias en la escuela primaria, aunque no hallan lo mismo en matemática.

Por su parte, Carrillo, Onofa y Ponce (2010) estudian el efecto de la incorporación de las TIC en el ambiente escolar respecto de los resultados educativos. Sobre la base de datos de la ciudad de Guayaquil, en el Ecuador, los autores analizan un programa de asistencia, por medio de computadoras, en la enseñanza de matemáticas y lenguaje en el nivel educativo primario. Realizan un diseño experimental y concluyen que las TIC tienen un impacto positivo en los resultados de matemáticas y estadísticamente no significativo en los de lenguaje. 
Cristia y otros (2012) estudian el impacto de "Una Laptop por Niño" en el Perú en escuelas del sector rural y de nivel primario y no encuentran evidencia de efectos en la tasa de escolarización, de deserción o repetición. Tampoco hallan efectos en los resultados educativos cognitivos en las áreas de lenguaje y matemática. Sin embargo, sí constatan un efecto positivo en las habilidades generales de los estudiantes.

En suma, en los trabajos de investigación citados hasta aquí se encuentra algún tipo de efecto positivo de las TIC en las competencias de los estudiantes. No obstante, hay investigaciones de las que se deducen conclusiones en sentido opuesto. Por ejemplo, Angrist y Lavy (2002) hallan que el uso de herramientas informáticas en los procesos de enseñanza-aprendizaje no tiene efectos relevantes en los logros educativos. Los autores llegan a esta conclusión luego de analizar en detalle el caso de Israel, donde se aplicó un programa de política focalizado en aumentar la disponibilidad de computadoras en una gran cantidad de escuelas a objeto de mejorar los resultados escolares.

Por su parte, Goolsbee y Guryan (2006) investigan el efecto de la aplicación de un programa de subsidio para el uso de Internet en las escuelas de California, en los Estados Unidos de América (denominado programa E-Rate). Estos autores utilizan una gran cantidad de variables para medir los resultados escolares y si bien demuestran que efectivamente aumentó el acceso a Internet en las aulas, no evidencian la existencia de una consecuencia de este cambio en el rendimiento escolar.

En estudios más recientes también se ha fallado en el intento de vincular las TIC con el rendimiento escolar. Por una parte, Sprietsma (2012) estima el efecto de la disponibilidad y uso de computadoras e Internet como herramientas pedagógicas en los resultados obtenidos por estudiantes brasileños de octavo grado en las pruebas de matemática y lectura, y — paradójicamente- halla una repercusión negativa de las TIC en el rendimiento escolar. Por otra parte, Witte y Rogge (2014) analizan el efecto de las Tic en el rendimiento académico de los estudiantes de Holanda utilizando datos del Estudio Internacional de Tendencias en Matemáticas y Ciencias (TIMSs) de 2011. Los autores aplican una metodología de emparejamiento y hallan que no existe una diferencia estadísticamente significativa entre los resultados obtenidos entre quienes disponen y hacen uso de la tecnología, y quienes no.

Sin embargo, Sprietsma (2012) señala que estos resultados desfavorables pueden explicarse haciendo hincapié en el tipo de uso que los alumnos le dan a la tecnología, la que puede representar un elemento de distracción más que un instrumento facilitador del aprendizaje. En igual sentido, otros autores también resaltan cuestiones vinculadas al uso de las TIC y hacen referencia a condicionantes que deben estar presentes para que su incorporación provoque efectos positivos en los logros académicos de los estudiantes. Entre ellos, Barrera-Osorio y Linden (2009) estudian el impacto del programa "Computadoras para la Educación" en Colombia y analizan el efecto del uso de las computadoras en el ámbito educativo por medio de un diseño experimental: algunas escuelas forman parte del programa, en tanto que otras no (grupo de control). Estos autores constatan que la incorporación de las computadoras no tiene un efecto en los resultados de aprendizaje y demuestran que ello se debe al mal uso que se hace de la tecnología. Si bien el programa provee asistencia técnica y entrenamiento a los docentes, los autores encuentran fallas en su puesta en marcha vinculadas a la falta de implementación de nuevas técnicas en las aulas por parte de los mismos docentes.

En igual sentido, Severín y otros (2011) analizan el impacto del programa "Una Laptop por Niño" en el Perú y su conclusión principal es que la tecnología es condición necesaria, pero no suficiente, para el logro de mejoras en los resultados educativos. Ellos sugieren que reviste gran relevancia fortalecer los instrumentos tecnológicos mediante elementos complementarios, así como el uso que se hace de la tecnología.

Por último, cabe mencionar los trabajos de Córdoba Gómez y Herrera Mejía (2013) y de Muñoz y Ortega (2014). Los primeros estudian la vinculación entre las TIC y el desempeño en matemáticas, y realizan un valioso aporte a la discusión acerca del efecto del uso de tecnologías en los resultados educativos. Con tal propósito, utilizan datos de estudiantes de dos instituciones educativas colombianas: una situada en el municipio de Medellín y otra en el de Duitama. Los autores concluyen que la incorporación de las tecnologías en el aula hace posible la obtención de mejores logros académicos solo si los docentes son acompañados por especialistas en el proceso de cambio de sus prácticas de enseñanza-aprendizaje.

Por su parte, Muñoz y Ortega (2014) analizan el impacto de dos planes llevados a cabo en Chile con el fin de incorporar el uso de las TIC en la enseñanza. Para ello, estudian los determinantes de los logros educativos cuantificados de acuerdo con las pruebas nacionales estandarizadas de aprendizaje, por medio de la metodología PSM. En términos generales, estos autores concluyen que los planes no han tenido efectos significativos en las puntuaciones de dichas pruebas, aunque sí observaron un impacto en los resultados de lenguaje en algunos grupos de estudiantes específicos, 
y estiman que es necesario establecer el desafío de optimizar la incorporación de las TIC en los procesos de enseñanza-aprendizaje, teniendo en cuenta otras variables que también afectan al rendimiento escolar.

En la Argentina no existe evidencia de estudios sobre los determinantes del rendimiento educativo que hayan centrado su atención en la variable de las TIC. Sin embargo, sí hay investigaciones que la han utilizado como control, encontrando que esta tiene un efecto estadísticamente significativo. Entre ellas, Santos (2007) detecta que la variable "computadoras por estudiante" tiene un papel positivo en el rendimiento escolar. Decándido

\section{IV}

\section{Metodología}

La relación entre la participación en el Programa Conectar Igualdad y el rendimiento educativo puede adolecer de endogeneidad. Es decir, las escuelas que participan del Programa podrían ser diferentes respecto de las que no participan, y tal diferencia podría estar correlacionada con el rendimiento educativo. Por este motivo, con el objetivo de estudiar el impacto que puede provocar el Programa Conectar Igualdad en el rendimiento educativo de los estudiantes de las escuelas medias, sería ideal disponer de un experimento donde la participación en el programa fuera independiente de ciertas características inherentes a la escuela. Sin embargo, la realización de un experimento de esta clase no resulta factible.

Por ello, el diseño cuasi experimental resulta adecuado cuando no se puede tener control sobre cómo se asigna la participación de un individuo en un grupo (en el caso aquí propuesto, en el grupo de beneficiarios del Programa Conectar Igualdad), ni sobre el resto de los factores bajo estudio.

Dado que la entrega de computadoras por medio del Programa es gradual, en el año 2012 algunas escuelas sí las habían recibido, mientras que otras no. En este contexto, el objetivo del presente trabajo consiste en dilucidar qué les hubiera sucedido - en términos de rendimiento educativo- a los estudiantes de las escuelas favorecidas con el Programa, si estas no hubieran participado en él. Entonces es necesario medir las mejoras en términos de rendimiento educativo de los estudiantes de las escuelas que participan en el Programa Conectar Igualdad, condicional al rendimiento anterior a la intervención, donde la mejora en el desempeño
(2011) halla que si el alumno realiza actividades en Internet sus logros son mayores. Formichella y Krüger (2013), Krüger (2013) y Formichella e Ibáñez (2014) evidencian un vínculo positivo entre la proporción de computadoras conectadas a Internet que posee la escuela (acceso a Internet) y el rendimiento escolar. Por último, Formichella e Ibáñez (2014) emplean un índice que representa los recursos vinculados a las TIC que posee el alumno en su hogar (incluye si tiene a disposición un software educativo, conexión a Internet y computadora) y encuentran una relación positiva entre dicho índice y el desempeño educativo. educativo sea la diferencia de los resultados obtenidos entre los estudiantes cubiertos por Conectar Igualdad y los no cubiertos. Una reconocida metodología que permite realizar el análisis descrito y es ampliamente utilizada en evaluación de impacto es la técnica de emparejamiento o PSM de Rosembaum y Rubin (1983), mediante la cual se construye artificialmente un "clon" o "match" para cada uno de los individuos estudiados con características idénticas, pero con una diferencia: la participación o no en el Programa Conectar Igualdad.

El PSM se puede resumir de la siguiente manera: i) se estima la probabilidad de que un estudiante reciba el tratamiento (participar del Programa Conectar Igualdad); la probabilidad predicha es el puntaje del estudiante; ii) se separa la muestra en dos submuestras: la de tratados (los que reciben el tratamiento) y la de controles (los que no reciben el tratamiento) y se ordenan ambas muestras de modo descendente, y iii) para cada tratado se busca un control con similar puntaje (probabilidad o verosimilitud) y se forman parejas (nótese que un mismo control puede ser emparejado con más de un tratado).

El siguiente paso es calcular la diferencia de los niveles de rendimiento educativo de cada pareja, para luego calcular la diferencia promedio en toda la muestra. Este resultado es conocido como el "efecto medio del tratamiento" en los tratados (ATE, por sus siglas en inglés). El error estándar de la diferencia entre cada pareja permite realizar una prueba t de significancia, para contrastar la hipótesis nula de ATE nulo. Si se rechaza la hipótesis, se puede asegurar que el ATE es estadísticamente diferente de cero, en caso contrario no lo es. 
En términos analíticos, se estima el efecto promedio de un tratamiento binario en un producto escalar continuo. Para un estudiante $i, \mathrm{i}=1, \ldots, \mathrm{N}$, con todas las unidades intercambiables, se define $\left(Y_{i}(0), Y_{i}(1)\right)$ como los dos productos potenciales, de manera tal que $Y_{i}(0)$ es el rendimiento educativo del estudiante $i$ cuando este no participa del Programa Conectar Igualdad y $Y_{i}(1)$ es el rendimiento educativo del estudiante i cuando está expuesto al tratamiento. El rendimiento educativo puede ser medido mediante los valores obtenidos por los alumnos en alguna prueba estandarizada de aprendizaje (PEA).

Si tanto el rendimiento educativo cuando el estudiante participa del Programa Conectar Igualdad, $Y_{i}(1)$, como cuando no participa, $Y_{i}(0)$, fueran observables, el efecto de la participación en el Programa (tratamiento) en el estudiante $i$ sería la diferencia $Y_{i}(1)-Y_{i}(0)$. El problema surge porque solo uno de estos productos es observable.

$$
Y_{i}=Y_{i}\left(W_{i}\right)=\left\{\begin{array}{l}
Y_{i}(0) \text { if } D_{i}=0 \\
Y_{i}(1) \text { if } D_{i}=1
\end{array}\right.
$$

donde $D_{i}$ indica si participa o no del Programa Conectar Igualdad. Según los modelos de Roy (1957), Quandt
(1972) y Rubin (1978), se asume que los rendimientos educativos de los estudiantes serían:

$$
\begin{aligned}
& Y_{1}=\mu_{1}(X)+U_{1} \\
& Y_{0}=\mu_{0}(X)+U_{0}
\end{aligned}
$$

La ganancia en términos de rendimiento educativo está dada por $\Delta=Y_{1}-Y_{0}$. Si $Y_{1}$ e $Y_{0}$ fueran observables para cada estudiante, la evaluación de impacto del tratamiento (participación en el Programa Conectar Igualdad) no sería un problema. Sin embargo, no ocurre usualmente que ambos estados sean observados al mismo tiempo para un estudiante. A fin de resolver este problema, se evalúan las políticas usando diferentes versiones de medias de variaciones sobre la población bajo estudio. Uno de los métodos es el "efecto medio del tratamiento" en los casos tratados (ATE).

Una comparación de los promedios de los resultados educativos según se participa o no en el Programa, nos dice algo acerca de los rendimientos potenciales, aunque no necesariamente explica el fenómeno. La comparación de los desempeños educativos promedio, considerando la condición "participación en el Programa", está formalmente relacionada con el efecto causal promedio dado por la siguiente ecuación:

$$
\underbrace{E\left(Y_{i} \mid D_{i}=1\right)-E\left(Y_{i} \mid D_{i}=0\right)}_{\begin{array}{c}
\text { Diferencia observada en } \\
\text { los rendimientos educativos } \\
\text { promedio }
\end{array}}=\underbrace{\left[E\left(Y_{1 i} \mid D_{i}=1\right)-E\left(Y_{0 i} \mid D_{i}=1\right)\right]}_{\begin{array}{c}
\text { ATT: efecto promedio de } \\
\text { participar en el Programa } \\
\text { Conectar en los tratados }
\end{array}}+\underbrace{\left[E\left(Y_{0 i} \mid D_{i}=1\right)-E\left(Y_{0 i} \mid D_{i}=0\right)\right]}
$$

En el contexto de tratamiento de los tratados, el problema surge porque en los datos usualmente no se incluyen observaciones de rendimiento educativo $Y_{0}$ para los estudiantes que participan del programa $(\mathrm{D}=1)$. El problema de sesgo de selección (Heckman, 1990) aparece como resultado de esta falta de información. El método de emparejamiento resuelve el problema de sesgo de selección reemplazando la aleatorización por el condicionamiento de los regresores. El sesgo de selección es eliminado solo si el tratamiento ha sido puramente aleatorio entre los estudiantes que tienen el mismo PSM.

La realización del emparejamiento mediante el PSM sirve para reducir el sesgo de selección, lo que permite la estimación de efectos de tratamiento con datos observables. Para este fin, se realiza una estimación de un modelo logit o probit, donde lo más relevante es la función de máxima verosimilitud, más que la significatividad de los estimadores (Heckman, Lalonde y Smith, 1999).

Existen diferentes métodos para estimar el impacto sobre el "efecto de tratamiento en los tratados" basados en el PSM, que se diferencian básicamente por la forma en que definen la distancia entre el tratado y el control, de los cuales se destacan:

i) Vecino más cercano (nearest neighbor matching) que empareja estudiantes tratados y de control, tomando una unidad tratada para cada unidad de control de acuerdo con el PSM más cercano. Se elige un estudiante no tratado $j$ para ser el contractual del estudiante $i$, de manera que, formalmente, el grupo de control del estudiante $i, \mathrm{C}^{\mathrm{O}}$ (pi) con PSM pi es solo un estudiante $j$ que cumple: $\mathrm{C}(\mathrm{i})=\min \mathrm{j}\left\|\mathrm{P}_{i}-\mathrm{P}_{j}\right\|$. 
El estudiante de control $j$ elegido del grupo de control es el que minimiza la diferencia entre su propensity score (PS) y el propensity score (PS) del estudiante tratado. En este estimador se emplea solo un estudiante del grupo de control (los que no participan del Programa Conectar Igualdad) para comparar con cada estudiante del grupo de tratamiento. En este caso, "el efecto promedio del tratamiento en los tratados (ATT, por sus siglas en inglés) se denomina ATTND (N por nearest neighbor o vecino más cercano).

ii) Estimador de Kernel, según el cual los estudiantes tratados (es decir, que recibieron el netbook) son emparejados con un promedio ponderado de todos los estudiantes de control con ponderaciones que son inversamente proporcionales a la distancia entre el PSM de los estudiantes tratados y el PSM de los estudiantes de control. En este caso, el ATT se denomina ATTK (K por Kernel).

iii) Estratificación: permite realizar un emparejamiento entre casos y controles basado en una variable que contiene el número de bloque (estrato) al que pertenece el registro de la zona de soporte común. La región de soporte común implica considerar en la estimación del "efecto promedio del tratamiento en los tratados (ATT) a los estudiantes que pertenecen al rango delimitado por los PS (propensy score) mínimo y máximo de los estudiantes del grupo de tratamiento. De esta manera, se asegura que la región definida cuente con valores con densidad positiva tanto para los estudiantes tratados como para los de control (Smith y Todd, 2005). En este caso, el ATT se denomina ATTS (s por stratification matching o emparejamiento por estratificación).

\section{Datos}

Se utiliza información del Programa Internacional de Evaluación de Estudiantes (PISA), que es elaborado cada tres años por la oCDE desde el año 2000 y cuyo objetivo es medir las competencias de los estudiantes de 15 años de diferentes países. El Programa PISA comprende las áreas de Matemática, Ciencias y Lenguaje, y en cada oportunidad se elige una de estas como prioritaria de forma rotativa. Sin embargo, PISA recopila también información acerca del contexto social de los estudiantes y de las escuelas a las que estos asisten (OCDE, 2009).

La Argentina participó en las pruebas PISA en los años 2000, 2006, 2009 y 2012. En el presente trabajo se emplean los datos correspondientes al año 2012 por ser los más actuales y contener información posterior a la implementación del Programa Conectar Igualdad, foco de interés del presente trabajo ${ }^{2}$.

La escala de puntuaciones de las pruebas PISA se confecciona de manera tal que la media es 500 y el desvío estándar 100. Los resultados de las evaluaciones son presentados utilizando "valores plausibles" (PV), que son una representación del conjunto de capacidades en un estudiante. Dado que el objetivo de PISA es evaluar las destrezas de una población y no de cada individuo en particular, cada alumno responde a un cierto número de ítems y se estima cómo hubiera contestado en todos los casos. Debido a ello, el equipo de PISA elabora cinco valores plausibles para cada área a partir de la información obtenida (OCDE, 2009).

Por tal motivo, el método correcto para estimar consistentemente cualquier valor estadístico $\mathrm{y}$, por ende, cualquier parámetro de un modelo consiste en realizar cálculos por separado con cada uno de estos cinco valores y luego obtener su promedio (OCDE, 2009). En consecuencia, dicho procedimiento es el que se ha tenido en cuenta en este trabajo.

\section{Variables}

La principal variable de interés de este trabajo se denomina Conectar y es una variable binaria que toma valor 1 si el estudiante asiste a una escuela que ha sido cubierta por el Programa Conectar Igualdad y cero en caso contrario. Es decir, se asigna el tratamiento a aquellos estudiantes de escuelas que en 2012 participaron en dicho Programa. Con este fin, se tuvo en cuenta el identificador de escuela (SCHOOLID) y de estudiante (STIDSTD).

Dado que la Argentina no ha participado en el módulo especial de las TIC disponible en la Prueba PISA, la base de datos no provee información acerca de qué escuelas están participando del Programa Conectar Igualdad y cuáles no. Por lo tanto, en este trabajo se construye la variable Conectar por medio de inferencias.

Como ya se ha mencionado, el Programa solo da cobertura a las escuelas de gestión pública; por ende, la variable toma valor cero en todos los casos de alumnos que asisten a escuelas de gestión privada. Con respecto a

\footnotetext{
${ }^{2}$ Es preciso aclarar que no es posible conocer el rendimiento educativo por alumno a lo largo del tiempo, ya que la base de PISA, al considerar únicamente a los estudiantes de 15 años escolarizados, se renueva período a período y solo se dispone de información de corte transversal. Por otra parte, dado que es imposible conocer el rendimiento educativo de una misma persona en ambos estados (con el Programa Conectar Igualdad y sin él) en el mismo período de tiempo, se justifica el uso de la metodología de emparejamiento. Esta, precisamente, permite comparar artificialmente el rendimiento en ambos estados.
} 
las escuelas de gestión pública, se utilizan otras variables para concluir si el colegio ya ha sido o no cubierto por el Programa.

A partir del cuestionario que responden los directores, se toman en cuenta dos preguntas:

i) Cantidad de alumnos en la franja etaria de 15 años (SC11Q01).

ii) Cantidad de computadoras disponibles con fines educativos para los alumnos de 15 años (SC11Q02).

A partir de estas variables, se calcula el cuociente entre la cantidad de computadoras disponibles y el total de alumnos (computadoras por alumno). Se supone que aquellas escuelas públicas —donde al menos el 95\% de sus alumnos de 15 años cuentan con computadoraparticipan en el Programa Conectar Igualdad ${ }^{3}$.

La deducción anterior cobra mayor sentido al observar la misma información para el año 2009, período previo a la implementación del Programa. En este caso, tan solo un $1,5 \%$ de los estudiantes que asisten a escuelas de gestión pública cumplen con la condición mencionada. Mientras que en el año 2012, transcurridos dos años de ejecución del Programa, dicho porcentaje es del 19,9\%, es decir, que registra un aumento de 18 puntos porcentuales, lo que implica una variación porcentual positiva igual a $12 \% 4$.

Como se describió en la sección II de este artículo, el Programa Conectar Igualdad prevé el uso de las netbooks no solo en la escuela, sino también en el hogar; por consiguiente, los estudiantes pueden llevar el equipo a sus casas para la elaboración de las tareas escolares. La encuesta PISA posee información con respecto a la disponibilidad de computadoras en el hogar. Participan en el Programa Conectar Igualdad los estudiantes cuyos establecimientos educacionales proporcionan computadoras al menos al $95 \%$ de sus

\footnotetext{
${ }^{3}$ Si bien en el Programa se prevé que la totalidad de los alumnos accedan a una computadora para estudiar en su escuela (lo que implicaría considerar solo a aquellas escuelas que poseen la razón descrita con un valor igual o mayor al 100\%), se decidió relajar esta condición (95\%). Esta decisión se tomó por recomendación de informantes clave que resaltaron la existencia de cuestiones operativas causantes de que una pequeña fracción de alumnos que asisten a escuelas cubiertas por el Programa no dispongan de una computadora.

4 Asimismo, la deducción cobra mayor relevancia al observar lo sucedido en las escuelas de gestión privada. En este sector, los estudiantes que cumplen con la condición establecida representan el $9 \%$ en 2009 y el $22 \%$ en 2012, es decir, existen 13 puntos porcentuales de diferencia entre los años considerados, lo que constituye tan solo un $1,4 \%$ en términos de variación porcentual. En suma, el aumento de la proporción de los estudiantes que cumplen con la condición que se puede observar a nivel global (de 4,5\% a 15,9\%), se debe sobre todo al cambio ocurrido en el sector de gestión pública por intermedio del Programa Conectar Igualdad.
}

alumnos y aquellos que disponen de computadora en el hogar (en el supuesto de que se cumplen las condiciones del Programa).

Por otra parte, las variables consideradas para calcular la probabilidad de participar en el Programa son:

- Repetidor: variable dicotómica que toma valor $1 \mathrm{si}$ el alumno ha repetido algún grado en la primaria o secundaria.

- Estudios secundarios: variable dicotómica que toma valor 1 si el nivel educativo más alto de los padres es el bachillerato o los ciclos formativos (grado medio o grado superior).

- Estudios terciarios: variable dicotómica que toma valor 1 si el nivel educativo más alto de los padres es el universitario.

- Auto2: variable dicotómica que toma valor 1 si la familia del alumno posee dos o más automóviles.

- Desempleado: variable dicotómica que toma valor 1 si el padre o la madre de la familia del alumno se encuentra sin empleo.

- Urbana: variable dicotómica que toma valor 1 si la escuela se ubica en una localidad con población mayor o igual a 15 mil habitantes y 0 en caso contrario.

- Promedioescs: variable continua que refleja la composición social del alumnado. Se calcula como el promedio del índice de situación económica, social y cultural $\left(\operatorname{ESCS}^{5}\right)$ de la escuela. El indicador ESCS correspondiente a cada alumno es elaborado por el equipo de PISA de la OCDE y en él se resume la información sobre el estatus ocupacional de los padres, su nivel educativo y las posesiones materiales y culturales del hogar (OCDE, 2009).

El Escs se calcula utilizando otros índices también presentados por el equipo de PISA: máximo nivel ocupacional de los padres (HISEI), máximo nivel educativo de los padres (PARED) e índice de posesiones en el hogar (HOMEPOS). A continuación se detalla la información resumida en cada uno de los índices:

HISEI: representa el estatus ocupacional de los padres de los estudiantes y corresponde al mayor valor entre padre y madre.

PARED: representa el nivel educativo de los padres medido como la cantidad de años de estudio aprobados. Se determina como el nivel más alto entre padre y madre.

\footnotetext{
5 Este índice y el resto de los que se describen a continuación son construidos por el equipo de PISA, de modo tal que un valor positivo representa que el nivel del hogar se encuentra por sobre el promedio de los países de la OCDE, mientras que un valor negativo representa lo contrario.
} 
HOMEPOS: resume información sobre la cantidad de libros que hay en el hogar y los índices WEALTH, HEDRES y CULTPOS.

WEALTH representa las posesiones materiales existentes en la vivienda del estudiante, HEDRES indica la cantidad de recursos educativos y CULTPOS representa la cantidad de posesiones culturales del hogar.

- Internet_casa: variable dicotómica que toma valor 1 si el alumno posee servicio de Internet en su hogar.

- COMPWEB: variable continua que se define como la proporción de computadoras para fines educativos conectadas a Internet que hay en el establecimiento.

\section{Análisis descriptivo}

La muestra de PISA 2012 para la Argentina consta de 5.908 observaciones, de las cuales el 15,9\% corresponde a alumnos que asisten a escuelas cubiertas por el Programa Conectar Igualdad. Sin embargo, se decidió excluir del análisis a aquellos estudiantes que poseen computadora en su hogar, pero que no participan del Programa. Esta medida responde a que la estimación correcta del impacto del tratamiento en los tratados requiere que el rendimiento educativo en los controles deba ser independiente de la asignación del tratamiento. La disponibilidad de computadoras en el hogar podría afectar el rendimiento educativo de los controles.

El hecho de trabajar con submuestras ha sido observado en otras investigaciones (Dehejia y Wahba, 2002; Johar, 2009). Este último evalúa la efectividad de un programa nacional de salud en Indonesia. Con este fin, selecciona de la muestra solo a los individuos adultos (mayores de 15 años), dado que la salud de los niños sería muy difícil de interpretar. A medida que estos se vuelven adultos, la frecuencia de los controles regulares desciende naturalmente. Por otra parte, Johar (2009) estima el PSM para subgrupos (jefe de hogar, hijo y otros) con el objeto de que los grupos sean de características más comunes.

Sobre la base de los datos de PISA, se seleccionaron 1.922 estudiantes, de los cuales 938 participaron en el Programa Conectar Igualdad (véase el gráfico 2).

Como puede observarse en el cuadro 2, las notas de rendimiento promedio son mayores respecto del grupo que es beneficiario del Programa para las tres competencias evaluadas por PISA.

Sin embargo, el problema de realizar una simple comparación de promedios de los rendimientos educativos entre los estudiantes con y sin Conectar Igualdad es que el proceso de asignación del Programa no ha sido aleatorio. Por esta razón, tal diferencia en el rendimiento puede estar dada por las características propias de los estudiantes (individuales, escolares, sociodemográficas y otras) que hacen que ciertos individuos participen en el Programa Conectar Igualdad y no por la participación en sí misma. En el cuadro 3 se muestran las principales características tanto del grupo de estudiantes con Conectar como sin Conectar, antes de la estimación del emparejamiento. Los datos corresponden al año 2012 del Programa PISA.

Se puede observar que los estudiantes de escuelas que participan en el Programa Conectar Igualdad presentan, en promedio, un menor número de repetidores, una mayor proporción de padres con estudios terciarios, menor proporción de padres desempleados, mayor proporción de hogares con acceso a Internet, mayor proporción de computadoras con acceso a Internet en la escuela y mayor proporción de escuelas con nivel socioeconómico alto. Estas diferencias promedio entre ambos grupos son estadísticamente significativas según el análisis de la varianza (ANOVA). La diferencia promedio en la proporción de padres con estudios secundarios es la única no significativa.

GRÁFICO 2

\section{Estudiantes que participan en el Programa Conectar Igualdad}

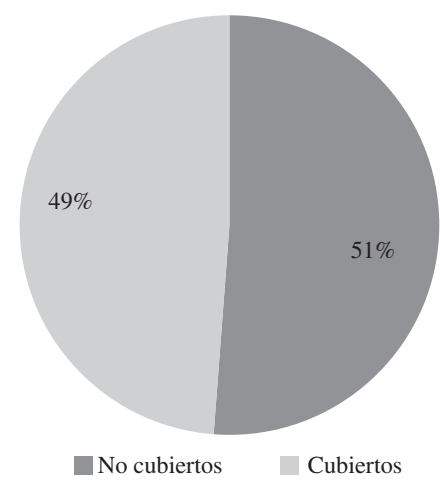

Fuente: Elaboración propia sobre la base de datos de la Prueba PISA 2012.

CUADRO 2

Diferencias de promedios de rendimiento educativo

\begin{tabular}{lccc}
\hline Competencias & $\begin{array}{c}\text { Con Conectar } \\
\text { Igualdad }\end{array}$ & $\begin{array}{c}\text { Sin Conectar } \\
\text { Igualdad }\end{array}$ & $\begin{array}{c}\text { Diferencia de } \\
\text { promedios }\end{array}$ \\
\hline Matemática & 397,033576 & 351,374268 & $45,6593081^{*}$ \\
Lectura & 402,013162 & 347,956902 & $54,056261^{*}$ \\
Ciencias & 409,325116 & 361,588903 & $47,736213^{*}$ \\
\hline
\end{tabular}

Fuente: Elaboración propia.

Nota: * estadísticamente significativa al $1 \%$. 
CUADRO 3

Estadísticos descriptivos

\begin{tabular}{|c|c|c|c|c|c|c|c|}
\hline Variable & Grupo & Observaciones & Promedio & $\begin{array}{l}\text { Desviación } \\
\text { estándar }\end{array}$ & Mínimo & Máximo & ANOVA \\
\hline \multirow[t]{2}{*}{ Repetidor } & $\mathrm{CC}$ & 750 & 0,21466 & 0,41085 & 0 & 1 & $* * *$ \\
\hline & $\mathrm{SC}$ & 654 & 0,39908 & 0,49005 & 0 & 1 & \\
\hline Estudios secundarios & $\mathrm{CC}$ & 906 & 0,20529 & 0,40412 & 0 & 1 & Ns \\
\hline \multirow[t]{2}{*}{ Estudios terciarios } & $\mathrm{CC}$ & 906 & 0,53200 & 0,49925 & 0 & 1 & $* * *$ \\
\hline & $\mathrm{SC}$ & 914 & 0,30087 & 0,45889 & 0 & 1 & \\
\hline \multirow[t]{2}{*}{ Auto2 } & $\mathrm{CC}$ & 891 & 0,18967 & 0,39224 & 0 & 1 & $* * *$ \\
\hline & $\mathrm{SC}$ & 877 & 0,08208 & 0,27461 & 0 & 1 & \\
\hline Desempleado & $\mathrm{SC}$ & 726 & 0,27415 & 0,44637 & 0 & 1 & \\
\hline \multirow[t]{2}{*}{ Urbana } & $\mathrm{CC}$ & 938 & 0,59483 & 0,49117 & 0 & 1 & $* * *$ \\
\hline & $\mathrm{SC}$ & 957 & 0,61651 & 0,48649 & 0 & 1 & \\
\hline \multirow{2}{*}{$\begin{array}{l}\text { Nivel socioeconómico } \\
\text { promedio }\end{array}$} & $\mathrm{CC}$ & 938 & $-0,55933$ & 0,71468 & $-1,8635$ & 1,075 & $* * *$ \\
\hline & $\mathrm{SC}$ & 984 & $-1,09734$ & 0,63107 & $-2,695$ & 1,075 & \\
\hline \multirow[t]{2}{*}{ Internet_casa } & $\mathrm{CC}$ & 884 & 0,85857 & 0,34864 & 0 & 1 & $* * *$ \\
\hline & $\mathrm{SC}$ & 845 & 0,19526 & 0,39664 & 0 & 1 & \\
\hline \multirow[t]{2}{*}{ COMPWEB } & $\mathrm{CC}$ & 851 & 0,90307 & 0,25583 & 0 & 1 & $* * *$ \\
\hline & $\mathrm{SC}$ & 885 & 0,93442 & 0,21881 & 0 & 1 & \\
\hline
\end{tabular}

Fuente: Elaboración propia.

Nota: cC: Con Conectar; sc: Sin Conectar. Auto2: Variable dicotómica que toma valor 1 si la familia del alumno posee dos o más automóviles; ANOVA: Análisis de la varianza; CompweB: Variable continua que se define como la proporción de computadoras para fines educativos conectadas a Internet que hay en el establecimiento; Ns = No significativa.

\section{V}

\section{Resultados}

En primer lugar, se estima el PSM usando un modelo probit que incluye las variables explicativas de la participación en el Programa Conectar Igualdad. En la estimación de la probabilidad solo se deben incluir variables que afecten a la decisión de participación y a la variable de resultado de manera simultánea (Bernal y Peña, 2011).

La variable dependiente es:

Conectar Igualdad: es una variable binaria que toma el valor $1 \mathrm{si} \mathrm{el} \mathrm{alumno} \mathrm{pertenece} \mathrm{a} \mathrm{una} \mathrm{escuela} \mathrm{que}$ ya está bajo la cobertura del Programa y cero en caso contrario. Se supone que si la escuela ha sido cubierta por el Programa, todos los alumnos poseen la computadora que se otorga mediante este.

El modelo probit empleado por el programa STATA 12 proviene de una variable latente o no observable del modelo, $\mathrm{y}^{*}$, que indica la propensión del estudiante a participar del Programa. Esta variable se explica a través de una serie de variables independientes observables mediante la siguiente ecuación estructural:

$$
y^{*}=\beta 0+x \beta+e \cdot y=1\left[y^{*}>0\right]
$$


La relación entre la variable observable binaria (si la escuela ha sido beneficiada por el Programa) y la variable latente $\mathrm{y}^{*}$ (la propensión a participar en el Programa) es representada por medio de la siguiente ecuación:

$$
\begin{aligned}
& y=1 \text { si } y^{*}>0 \\
& y=0 \text { si } y^{*} \leq 0
\end{aligned}
$$

Los estudios sobre implementación del Programa Conectar Igualdad, así como los trabajos sobre rendimiento educativo, brindan información respecto de los factores que inciden en la participación en él.

\section{Efecto de tratamiento: participación en el Programa Conectar Igualdad}

El objetivo consiste en identificar el efecto promedio de la participación en el Programa sobre el rendimiento educativo de los estudiantes de 15 años de la Argentina.

El PSM es válido si se cree que las variables observadas determinan la participación (participación en el Programa Conectar), es decir, si no hay sesgos por variables inobservables o si las variables no observables o no disponibles no sean un determinante fundamental tanto en la participación en el Programa como de las variables de resultado potenciales, tales como el rendimiento educativo (Bernal y Peña, 2011).

La estimación del emparejamiento usando sTATA 12 produce los resultados que pueden observarse en el cuadro 4. La bondad de ajuste del modelo es adecuada. El modelo en conjunto es significativo de acuerdo con el estadístico de razón de verosimilitud Razón (prob $>$ chi2=0,0000) y el Pseudo $\mathrm{R}^{2}$.

Posteriormente, se utiliza la técnica de emparejamiento para definir un soporte común. La región seleccionada ha sido [0,05431384, 0,9742593]. En el gráfico 3 se aprecia la densidad de Kernel del PSM estimado, en la que se puede apreciar la región de soporte común.

En el gráfico 3 se muestran dos funciones de densidad, una para el grupo de tratados (curva gris) y otra para el grupo de controles (curva negra). Esta función sugiere que el promedio de la probabilidad de participar en el Programa para el grupo de tratados es superior al promedio para el grupo de control.

De los 1.922 estudiantes de 15 años de la Argentina, se verifica que 949 de ellos pertenecen a la región de soporte común. Entre los estudiantes de esta región se encuentran 557 tratados y 392 controles (véase el cuadro 5).

Como se puede observar, el grupo de control es grande (392 estudiantes), lo que es una condición necesaria para la adecuación del método. Los resultados obtenidos indican que el nivel socioeconómico de la escuela, así como la disponibilidad de Internet en el hogar, poseen un efecto significativo y positivo en la probabilidad de participar en el Programa. Por otra parte, los estudiantes que son repetidores y cuyos padres están desempleados tienen significativamente menos probabilidades de participar en el Programa. Por su parte, la variable

CUADRO 4

\begin{tabular}{|c|c|c|c|}
\hline Variables & Coeficiente & Error estándar & Nivel de significatividad \\
\hline Repetidor & $-0,2720347$ & 0,1163132 & $* *$ \\
\hline Est._secundario & $-0,1118963$ & 0,139605 & Ns \\
\hline Est_terciario & 0,1032862 & 0,1257226 & Ns \\
\hline Auto2 & 0,1476435 & 0,1469592 & Ns \\
\hline Desempleado & $-0,2393344$ & 0,1326774 & $*$ \\
\hline Urbana & $-0,1304947$ & 0,1059158 & Ns \\
\hline Nivel socioeconómico promedio & 0,1754671 & 0,0850824 & $* *$ \\
\hline Internet_casa & 1,774768 & 0,1109653 & $* * *$ \\
\hline COMPWEB & $-0,4561941$ & 0,2155834 & $* *$ \\
\hline _Constante & $-0,0674548$ & 0,2647191 & Ns \\
\hline $\mathrm{N}^{\circ}$ de observaciones & 951 & & \\
\hline LR chi2 (9) & 495,93 & & \\
\hline Prob $>$ chi 2 & 0,0000 & & \\
\hline Pseudo $\mathrm{R}^{2}$ & 0,3844 & & \\
\hline
\end{tabular}

Determinantes de la participación en Programa Conectar Igualdad

Fuente: Elaboración propia.

Nota: $* * *$, ** significativo al $1 \%$ y $5 \%$ respectivamente; Ns = No significativa.

COMPWEB: Variable continua que se define como la proporción de computadoras para fines educativos conectadas a Internet que hay en el establecimiento; Auto2: Variable dicotómica que toma valor 1 si la familia del alumno posee dos o más automóviles. 
GRÁFICO 3

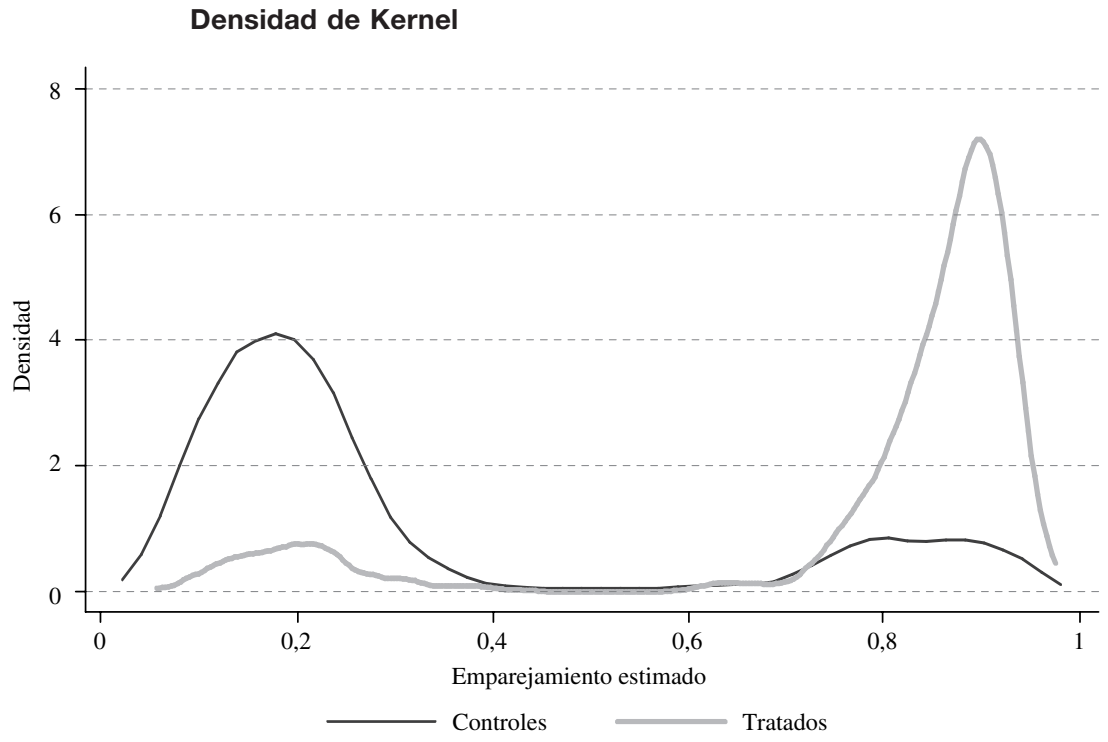

Tipo de función Kernel: Epanechnikov, ancho de banda: 0,0316.

Fuente: Elaboración propia.

CUADRO 5

\section{Distribución de los casos}

\begin{tabular}{lccc}
\hline Estudiantes & $\begin{array}{c}\mathrm{N}^{\mathrm{o}} \text { de } \\
\text { observaciones }\end{array}$ & Porcentaje & $\begin{array}{c}\text { Porcentaje } \\
\text { acumulado }\end{array}$ \\
\hline Control & 392 & 41,31 & 41,31 \\
Tratamiento & 557 & 58,69 & 100 \\
Total & 949 & 100 & \\
\hline
\end{tabular}

Fuente: Elaboración propia.

COMPWEB posee un efecto significativo y negativo, por lo que las escuelas con mayor proporción de computadoras con acceso a Internet tienen menos oportunidades de participar en el Programa.

Por lo tanto, en principio los establecimientos más necesitados — que no tienen disponibilidad de computadoras con acceso a Internet, su nivel socioeconómico promedio es más bajo, sus alumnos repetidores representan una mayor proporción y las familias con padres desocupados y sin Internet en casa son más frecuentes- poseen menos probabilidades de participar en el Programa ${ }^{6}$.

${ }^{6}$ Esta afirmación surge al examinar las variables que se han considerado para realizar el emparejamiento entre los alumnos y que resultaron ser estadísticamente significativas. El motivo por el cual las escuelas con condiciones socioeconómicas desfavorables no acceden al Programa
Posteriormente, se utiliza el emparejamiento estimado por el método de PSM. Las diferencias en los niveles de rendimiento educativo predichos por el modelo mediante las diferentes técnicas de emparejamiento para estimar el ATT se pueden observar en el cuadro 6: la diferencia en el rendimiento educativo promedio entre los estudiantes de escuelas beneficiadas con el Programa Conectar Igualdad y aquellos sin acceso a este es estadísticamente significativa de acuerdo con las técnicas de Estratificación y Kernel. Por el contrario, no hay evidencias estadísticamente significativas con la técnica del vecino más cercano.

Por consiguiente, se rechaza la hipótesis de que no existen diferencias en el rendimiento educativo entre los estudiantes tratados (que participan del Programa Conectar Igualdad) y los estudiantes de control. Si bien la diferencia en las pruebas es estadísticamente significativa, su cuantía promedio es baja. Este reducido impacto en el

o lo hacen con mayor demora, excede al objetivo del presente trabajo. Sin embargo, en función de comunicaciones con informantes clave se esboza aquí la siguiente hipótesis acerca de la causa. El proceso de ingreso en el Programa implica un pedido formal por parte de los directivos de las escuelas a la Administración Nacional de la Seguridad Social (ANSES) y los equipos de escuelas que atienden problemáticas sociales más difíciles disponen de menos tiempo y energía para realizar trámites. Esto no excluye a sus establecimientos del Programa, pero sí influye en que se incorporen con mayor lentitud que los colegios que poseen más recursos y albergan alumnos con mejores condiciones socioeconómicas. Esta hipótesis será objeto de investigaciones futuras. 
CUADRO 6

\section{Estimación del ATT en las pruebas}

\begin{tabular}{|c|c|c|c|c|c|}
\hline Prueba & PSM & Tratados & Controles & ATT & $\begin{array}{c}\text { Valor del estadístico } \\
\text { t de Student }\end{array}$ \\
\hline \multirow[t]{3}{*}{ Matemáticas } & ATTS & 557 & 392 & 18,9468 & 2,1874 \\
\hline & ATTK & 557 & 392 & 22,2852 & 2,9056 \\
\hline & ATTND & 557 & 135 & 11,1794 & 1,1474 \\
\hline \multirow[t]{3}{*}{ Lectura } & ATTS & 557 & 392 & 25,1496 & 2,6202 \\
\hline & ATTK & 557 & 392 & 28,8056 & 3,0984 \\
\hline & ATTND & 557 & 135 & 19,8906 & 1,6596 \\
\hline \multirow[t]{3}{*}{ Ciencia } & ATTS & 557 & 392 & 18,2304 & 1,7668 \\
\hline & ATTK & 557 & 392 & 20,7718 & 2,4338 \\
\hline & ATTND & 557 & 135 & 12,2406 & 1,245 \\
\hline
\end{tabular}

Fuente: Elaboración propia sobre la base de datos de la Prueba PISA 2012.

Nota: ATT: Tratamiento en los tratados; ATTK: Tratamiento de los tratados según Kernel; ATTND: Tratamiento de los tratados según Vecino más cercano; ATTS: Tratamiento en los tratados según estratificación; PSM: Propensity score matching.

rendimiento educativo constituye una evidencia de que la implementación del Programa, aún en estado incipiente, no ha explotado todas sus potencialidades. Tal como se especifica en el Programa, el uso apropiado de los netbooks exige que su posesión sea acompañada por el modo en que los docentes enseñan, incorporándolos en el proceso por medio del cual los estudiantes aprenden. Además, se requiere que la distribución de los equipos se complemente con la existencia de condiciones mínimas de acceso a Internet en las escuelas e implementación de estos equipos en ellas. Es decir, además de distribuirse los equipos, el Programa supone el acompañamiento de personal técnico capacitado, como también la formación de los docentes para su utilización con fines pedagógicos en las aulas.

Una prueba de que el programa aún no ha trascendido la etapa de mero acceso al equipo por parte de las escuelas, es que el impacto en el rendimiento educativo del Programa es muy similar al de la disponibilidad de computadoras en el hogar. A partir de la estimación con PSM del efecto de las computadoras en casa en el rendimiento de los estudiantes, se observan consecuencias en el "efecto medio del tratamiento" (ATE) muy similares a los obtenidos en el caso de la participación en el Programa Conectar Igualdad. Aunque las poblaciones bajo estudio son diferentes - ya que en este último caso se analizan todos los estudiantes de 15 años de la Argentina según datos provenientes de la Prueba PISA - se observan diferencias mínimas entre ambos casos (véase el cuadro 7) ${ }^{7}$.

Por último, siguiendo a Nannincini (2007) se puede demostrar que el modelo estimado es robusto, ya que se han especificado estimaciones del efecto promedio del tratamiento en los tratados (ATT) bajo diferentes escenarios posibles de desvío del supuesto de independencia condicional. Es decir, se pretende simular la capacidad de una variable no observable (no incluida en la estimación de la probabilidad) de generar sesgo. Con este fin, se realizaron estimaciones alternativas del ATT, incorporando variables binarias adicionales y ninguna arrojó diferencias en el ATT obtenido con el modelo. Tampoco se encontraron diferencias entre el impacto original y los impactos en el rendimiento educativo con la incorporación de variables adicionales.

\footnotetext{
7 Cabe recordar que el estudio presentado en este artículo se centra en una submuestra que asciende a 1.922 observaciones, mientras que el ejercicio propuesto que investiga el impacto de la variable computadora se basa en la muestra total de PISA 2012 para la Argentina. Asimismo, la presencia de alumnos que se benefician del Conectar Igualdad en ambas muestras no condiciona los resultados obtenidos en el ejercicio propuesto, debido a que la proporción que se encuentra en dicha situación es baja (18\%).
} 


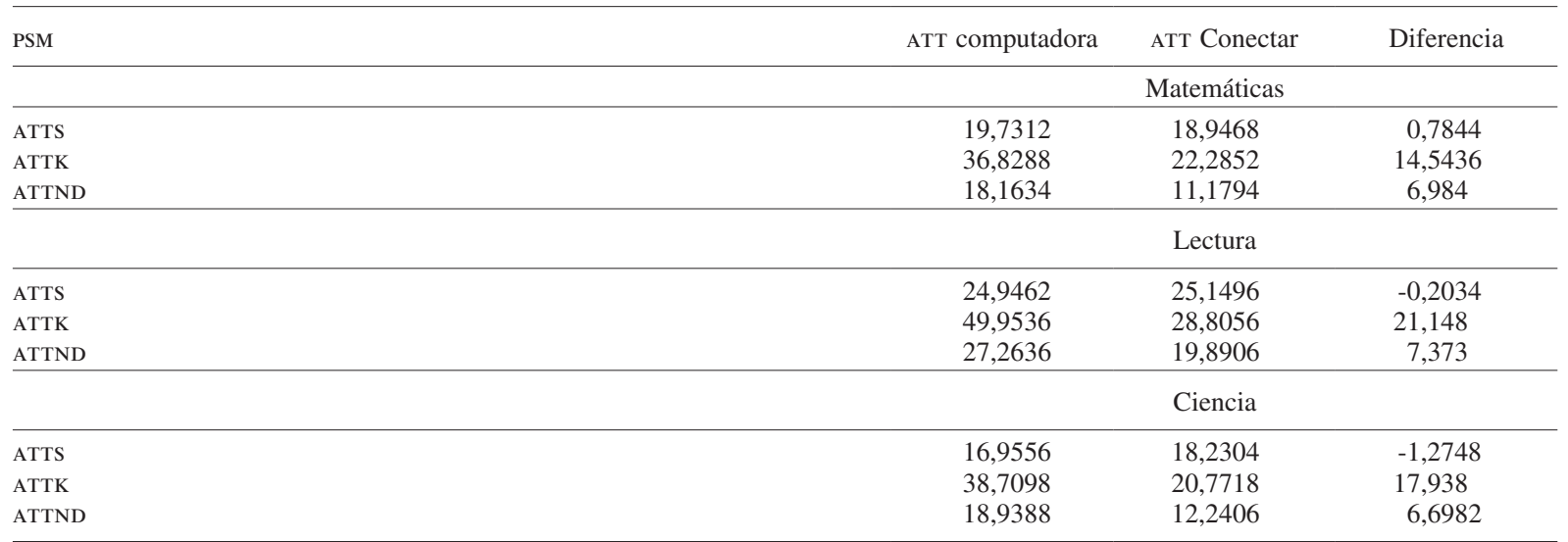

Fuente: Elaboración propia.

Nota: PSM: Propensity score matching; ATT: Tratamiento en los tratados; ATTK: Tratamiento de los tratados según Kernel; ATTND: Tratamiento de los tratados según Vecino más cercano; ATTs: Tratamiento en los tratados según estratificación.

\section{Consideraciones finales}

En el presente trabajo se ha analizado el papel de las TIC en la educación y, en particular, se ha estudiado su efecto como determinante del rendimiento educativo. Específicamente, se ha analizado el impacto del Programa Conectar Igualdad en la Argentina desde un punto de vista cuantitativo, con el fin de complementar los estudios de carácter cualitativo existentes hasta el momento y contribuir a un mejor entendimiento del uso y efecto de las TIC en el proceso de aprendizaje.

En resumen, mediante este artículo se han realizado dos aportes: se ha ampliado la base de conocimiento sobre el papel de las TIC en la educación y se ha evaluado el impacto del Programa Conectar Igualdad desarrollado en la Argentina.

Con respecto a los resultados -mediante el empleo de la técnica de emparejamiento o propensity score matching (PSM) con datos provenientes de PISA correspondientes al año 2012 para la Argentina-, se obtiene una diferencia estadísticamente significativa en el rendimiento educativo promedio entre el grupo de estudiantes beneficiados con el Programa y aquellos no beneficiados. Sin embargo, esta diferencia no necesariamente se traduce en un cambio cualitativo importante en términos de rendimiento educativo, ya que el valor absoluto de las diferencias de promedio es bajo. Por ese motivo, es de interés en futuras investigaciones explorar si la participación en el Programa Conectar Igualdad ha generado una disminución en el fracaso escolar.
A pesar de que no se dispone de información estadística explícita respecto de la participación de las escuelas en el Programa Conectar Igualdad, no se considera esta una limitación importante del trabajo dado que las variables utilizadas para corroborar la participación en él resultan coherentes. Tal coherencia se corrobora al comparar las estadísticas respecto del año 2009, previo a la implementación del Programa.

También se ha analizado el efecto que tienen las TIC en el rendimiento educativo, utilizando como variable de tratamiento la disponibilidad de computadoras en el hogar de los estudiantes. A partir de allí, se observa que la participación en Programa, cuya implementación tiene un alcance superior a la mera disponibilidad de computadoras, arroja resultados similares en términos de rendimiento educativo. Esto demostraría la necesidad de explotar las potencialidades del Programa, dado que hasta el momento sus resultados no trascienden los del acceso a las computadoras.

En la mayoría de los estudios se utilizan indicadores débiles de uso de las TIC para analizar su efecto en la educación. Entre los indicadores tradicionales sobre las TIC en las escuelas se encuentra el número de computadoras disponibles.

Finalmente, cabe señalar que el estudio de las Tic en la educación se encuentra en una etapa de desarrollo, por ello resulta necesario profundizar en la discusión acerca de esta. Asimismo, la investigación sobre el impacto de las políticas públicas tendientes a la universalización de las TIC requiere un seguimiento en el tiempo, que acompañe la evolución de los efectos de la implementación del programa. 


\section{Bibliografía}

Angrist, J. y V. Lavy (2002), "New evidence on classroom computers and pupil learning", The Economic Journal, vol. 112, N ${ }^{\circ} 482$, Royal Economic Society.

ANSES (Administración Nacional de la Seguridad Social) (2014), "La ANSES ya entregó casi cuatro millones de netbooks a traves de Conectar Igualdad" [en línea] www.conectarigualdad.gob.ar.

Aristizabal, G., M. Caicedo y D. Escandón (2009), "Las tecnologías de la información y comunicación como determinante en el rendimiento académico escolar, Colombia 2006-2009" [en línea] http://2012.economicsofeducation.com.

Barrera-Osorio, F. y L. Linden (2009), "The use and misuse of computers in education. Evidence from a randomized experiment in Colombia", Policy Research Working Paper, $\mathrm{N}^{\circ} 4836$, Washington, D.C., Banco Mundial.

Becta (2007), "Inclusive Learning: an Essential Guide" [en línea] http://www.tes.co.uk/teachingresource/Inclusive-learning-anessential-guide-6072357/.

Bernal, R. y X. Peña (2011), Guía práctica para la evaluación de impacto, Santiago, Ediciones UC.

Carneiro, R., J.C. Toscano y T. Díaz (2009), Los desafíos de las TIC para el cambio educativo, Madrid, Organización de Estados Iberoamericanos para la Educación, la Ciencia y la Cultura (OEI)-Fundación Santillana.

Carrillo, P., M. Onofa y J. Ponce (2010), "Information technology and student achievement: evidence from a randomized experiment in Ecuador", IDB Working Paper, $\mathrm{N}^{\circ}$ 223, Washington, D.C., Banco Interamericano de Desarrollo [en línea] http://www.iadb. org/res/publications/pubfiles/pubIDB-WP-223.pdf.

Castells, M. (1999), La era de la información. Economía, sociedad y cultura, Madrid, Siglo XXI.

Claro, M. (2011), "El papel de las tecnologías de la información y las comunicaciones en la educación inclusiva", Documento de proyecto (LC/W.434), Santiago, Comisión Económica para América Latina y el Caribe (CEPAL).

Claro, M. y otros (2011), "Aporte del sistema educativo a la reducción de las brechas digitales. Una mirada desde las mediciones PISA", Documento de Proyecto (LC/W.456), Santiago, Comisión Económica para América Latina y el Caribe (CEPAL).

Coleman, J. y otros (1966), Equality of Educational Opportunity, Washington, D.C., United States Government Publishing Office.

Consejo Federal de Educación (2010), "Las políticas de inclusión digital educativa. El Programa Conectar Igualdad" [en línea] http://www.me.gov.ar/consejo/resoluciones/res10/123-10_01. pdf.

Córdoba Gómez, F. y H. Herrera Mejía (2013), "Impacto del uso de objetos de aprendizaje en el desempeño en matemáticas de estudiantes de grado noveno", Revista Virtual Universidad Católica del Norte, $\mathrm{N}^{\circ} 39$.

Cristia, J. y otros (2012), "Technology and child development: evidence from the one laptop per child program", IDB Working Paper, $\mathrm{N}^{\circ} 63438$ [en línea] http://www.iadb.org/en/researchand-data.

Decándido, G. (2011), "Factores que afectan las competencias de los alumnos argentinos en PISA 2009. Un estudio empírico de dos niveles con efectos de interacción", Anales de la Asociación Argentina de Economía Política [en línea] http:// www.aaep.org.ar.

Dehejia, R. y S. Wahba (2002), "Propensity score matching methods for non experimental causal studies", The Review of Economics and Statistics, vol. 84, $\mathrm{N}^{\circ} 1$, Cambridge, Massachusetts, The MIT Press.

Formichella, M.M. (2011), “Se debe el mayor rendimiento de las escuelas de gestión privada en la Argentina al tipo de administración?", Revista CEPAL, $\mathrm{N}^{\circ} 105$ (LC/G.2508-P), Santiago, Comisión Económica para América Latina y el Caribe (CEPAL).
Formichella, M.M. y M. Ibáñez (2014), "Género e inequidad educativa: un análisis para el nivel medio en Argentina", Estudios Económicos Regionales y Sectoriales, vol. 14, $\mathrm{N}^{\circ} 1$.

Formichella, M.M. y N. Krüger (2013), "El fracaso escolar en el nivel medio argentino: ies menos frecuente en las escuelas de gestión privada debido a su administración?", Estudios económicos regionales y sectoriales, vol. $13, \mathrm{~N}^{\circ} 3$.

Goolsbee, A. y J. Guryan (2006), "The impact of internet subsidies in public schools", The Review of Economics and Statistics, vol. 88, $\mathrm{N}^{\circ}$ 2, Cambridge, Massachusetts, The MIT Press.

Hargittai, E. (2002), "Second-level digital divide: difference in peoples online skills", First Monday, vol. 7, 우 4 [en línea] http://firstmonday.org/htbin/cgiwrap/bin/ojs/index.php/fm/ article/view/942/864.

Heckman, J. (1990), "Varieties of selection bias", American Economic Review, vol. 80, $\mathrm{N}^{\circ}$ 2, Nashville, Tennessee, American Economic Association.

Heckman, J., R. Lalonde y J. Smith (1999), "The economics and econometrics of active labor market programs", Handbook of Labor Economics, vol. 3, parte A, O. Ashenfelter y D. Card (eds.), Amsterdam, North-Holland.

Johar, M. (2009), "The impact of the Indonesian health card program: a matching estimator approach", Journal of Health Economics, vol. 28, $\mathrm{N}^{\circ} 1$, Amsterdam, Elsevier.

Kozma R.B. (2008), "Comparative analysis of policies for ICT in education", International Handbook on Information Technology in Primary and Secondary Education, J. Voogt y G. Knezek (eds.), Nueva York, Springer.

(ed.) (2003), Technology, Innovation and Educational Change: A Global Perspective, Eugene, International Society for Technology in Education.

Krüger, N. (2013), "Segregación social y desigualdad de logros educativos en Argentina", Archivos Analíticos de Políticas Educativas, vol. 21, No 86 [en línea] http://epaa.asu.edu/ojs/ article/view/1352.

Lara, P. y J.M. Duart (2005), "Gestión de contenidos en el e-learning: acceso y uso de objetos de información como recurso estratégico", Revista de Universidad y Sociedad del Conocimiento, vol. 2, $\mathrm{N}^{\circ} 2$ [en línea] http://www.uoc.edu/ rusc/2/2/dt/esp/lara.pdf.

Llach, J., S. Montoya y F. Roldan (1999), Educación para todos, Buenos Aires, IERAL.

Machin, S., S. McNally y O. Silva (2006), "New technology in schools: is there a payoff?", IZA Discussion Paper, $\mathrm{N}^{\circ} 2234$ [en línea] http://ftp.iza.org/dp2234.pdf.

Maguire, C. y J. Zhang (2007), "Blended learning in the development context. Experience with GDLN in Asia-Pacific", Tokyo Development Learning Center [en línea] https://www. jointokyo.org/files/cms/news/pdf/Blended_Learning_in_the_ Development_Context_v1.pdf

Muñoz, R. y J. Ortega (2014), “'Tienen la banda ancha y las TICs un impacto positivo sobre el rendimiento escolar? Evidencia para Chile", documento presentado en la octava conferencia CPR LATAM Conference, Bogotá

Nannincini, T. (2007), "Simulation-based sensitivity analisys for matching estimators", Stata Journal, vol. 7, No 3

oCDE (Organización para la Cooperación y el Desarrollo Económicos) (2009), PISA Data Analysis Manual, París, OECD Publishing.

Parshall, C.G. y otros (2002), Practical Considerations in Computerbased Testing, Nueva York, Springer.

Robinson, J.P, P. DiMaggio y E. Hargittai (2003), "New social survey perspectives on the digital divide", IT\&Society, vol. $1, \mathrm{~N}^{\circ} 5$.

Rodríguez, P., M. Nussbaum y L. Dombrovskaia (2013), "ICT for education: a conceptual framweork for the sustainable adoption of technology enhanced learning environments in schools", Technology, Pedagogy and Education, vol. 21, $\mathrm{N}^{\circ} 3$, Taylor \& Francis. 
Rosenbaum, P.R. y D.B. Rubin (1983), "The central role of the propensity score in observational studies for causal effects", Biometrika, vol. 70, $\mathrm{N}^{\circ}$ 1, Oxford University Press.

Santos, M. (2007), "Quality of education in Argentina: determinants and distribution using PISA 2000 test scores", Well-Being and Social Policy, vol. 3, $\mathrm{N}^{\circ} 1$.

Selwyn, N. (2004), "Reconsidering political and popular understandings of the digital divide", New Media \& Society, vol. $6, \mathrm{~N}^{\circ} 3$, SAGE.

Severín, E. y otros (2011), Evaluación del programa "una laptop por niño" en Perú: resultados y perspectivas, Washington, D.C., Banco Interamericano de Desarrollo.

Siteal (Sistema de Información de Tendencias Educativas en América Latina) (2014), Informe sobre tendencias sociales y educativas en América Latina 2014. Políticas TIC en los sistemas educativos de América Latina, París, Organización de las Naciones Unidas para la Educación, la Ciencia y la Cultura (UNESCO)/Organización de Estados Iberoamericanos para la Educación, la Ciencia y la Cultura (OEI) [en línea] http://www.siteal.org/sites/default/files/siteal_informe_2014_ politicas_tic.pdf.

Smith, J.A. y P.E. Todd (2005), "Does matching overcome LaLonde's critique of nonexperimental estimators?", Journal of Econometrics, vol. 125, $\mathrm{N}^{\circ} 1-2$, Amsterdam, Elsevier.

Spiezia, V. (2010), "Does computer use increase educational achievements? Student-level evidence from PISA", OECD Journal: Economic Studies, vol. 2010 [en línea] http://www1. oecd.org/eco/labour/49849896.pdf.

Sprietsma, M. (2012), "Computers as pedagogical tools in Brazil: a pseudo-panel analysis", ZEW Discussion Papers, 07-040 [en línea] http://hdl.handle.net/10419/24604.

Sunkel, G. (2006), "Las tecnologías de la información y la comunicación (TIC) en la educación en América Latina. Una exploración de indicadores", serie Políticas Sociales, $\mathrm{N}^{\circ} 126$ (LC/L.2638-P), Santiago, Comisión Económica para América Latina y el Caribe (CEPAL).
Sunkel, G. y D. Trucco (eds.) (2012), Las tecnologías digitales frente a los desafios de una Educación Inclusiva en América Latina: algunos casos de buenas prácticas (LC/L.3545), Santiago, Naciones Unidas.

(2010), "Nuevas tecnologías de la información y la comunicación para la educación en América Latina: riesgos y oportunidades", Serie Políticas Sociales, $\mathrm{N}^{\circ} 167$ (LC/ L.3266-P), Santiago, Comisión Económica para América Latina y el Caribe (CEPAL).

Sunkel, G., D. Trucco y S. Möller (2011), "Aprender y enseñar con las tecnologías de la información y las comunicaciones (TIC) en América Latina. Potenciales beneficios", serie Políticas Sociales, № 169 (LC/L.3291-P), Santiago, Comisión Económica para América Latina y el Caribe (CEPAL).

Terzis, V. y A. Economides (2011), "The acceptance and use of computer based assessment", Computers \& Education, vol. 56, $\mathrm{N}^{\circ} 4$, Amsterdam, Elsevier.

UNESCO (Organización de las Naciones Unidas para la Educación, la Ciencia y la Cultura) (2014), Reading in the Mobile Era. A Study of Mobile Reading in Developing Countries, París.

UNICEF (Fondo de las Naciones Unidas para la Infancia) (2014a), Las políticas TIC en los sistemas educativos de América Latina. Caso Brasil. Programa TIC y Educación Básica, Buenos Aires. (2014b), Las politicas TIC en los sistemas educativos de América Latina. Caso Colombia. Programa TIC y Educación Básica, Buenos Aires.

(2013a), Las políticas TIC en los sistemas educativos de América Latina: caso argentino, Buenos Aires.

(2013b), Las políticas TIC en los sistemas educativos de América Latina. Caso Chile. Programa TIC y Educación Básica, Buenos Aires.

(2013c), Las políticas TIC en los sistemas educativos de América Latina. Caso Perú. Programa TIC y Educación Básica, Buenos Aires.

Witte, K.N. y N. Rogge (2014), "Does ICT matter for effectiveness and efficiency in mathematics education?", Computers \& Education, vol. 75, Amsterdam, Elsevier. 